Aims With timely diagnosis and treatment of urinary infections impairment of the renal (kidney) function is prevented.

Materials and methods Data from 610 children aged 0 to 18 years suspected of urinary infection or febrile for a period of 3 years (2011-2013) had been processed. Analytical and descriptive method had been used for processing.

Results Out of 320 children with taken urine - culture, 102 showed positive results $(31,87 \%)$. Escherichia coli had been isolated with 84 children (82,35\%), Proteus sp. with 7 children $(6,86 \%)$, Klebsiella with children 5 (4,91\%), Pseudomonas aeruginosa had been isolated with 4 children $(3,92 \%)$ and other bacteria with 2 children (1,96\%). From the lab findings the most common had been leucocytosis. One child had been diagnosed agenesis of the right kidney, 6 children had been diagnosed VUR, and 1 child ectopic kidney. During the treatment the following drugs had been used: Nitrofurantoin, Amoxicillin + clavulonic acid, Trimetoprim + sulfonamide, Cefuroxim or Cefixime depending on the antibiogram. It resulted in the negative urineculture in more than $95 \%$ of children, while children with congenital malformations are under regular scrutiny by the paediatrician - nephrologists.

Conclusion Routine investigation of urine during every obscure febrile condition. Urine- culture method is necessary to determine the specific therapy.

Timely diagnosis and treatment leads to successful prevention of renal (kidney) damage.

\section{P0-0796 WITHDRAWN}

\section{PO-0797 EVALUATION OF PATIENTS WITH DIAGNOSIS OF UROLITHIASIS IN UMRANIYE REGION OF ISTANBUL}

G Yildirim Usta, H Tekkus, A Yazar, S Akova, R Gurpinar, B Karakayali, S Guven, I Islek. Pedatrics, Umraniye Training and Research Hospital, Istanbul, Turkey

\subsection{6/archdischild-2014-307384.1432}

Urinary tract stones (nefrolithiasis, urolithiasis) is an endemic disease, which is seen in developing countries including our country. In this study, our aim was to evoluate the patients with the diagnosis of urolithiasis retrospectively, between the years of 2013 to 2014 . On this purpose, 80 patients diagnosed with urolithiasis were evaluated retrospectively.

There was not any statistically significant difference between the patients evaluated by means on sex (50\% were female). $62,5 \%$ of the patients were under the age of five years. Positive family history for urolithiasis was determined in $37.5 \%$ of the patients. Renal stones were detected on the left kidney in $47,5 \%$ of the patients. Full urinalysis showed hematuria in $32,5 \%$ of the patients, pyuria in $27,5 \%$, proteinuria in $13.75 \%$ respectively. The mostly seen metabolic disorder was hiperoxaluria with 25\% (also hiperuricasiduria was seen in 21\%, and hipercalciuria in $10 \%)$. The patient were taken under control with medical treatment and metaphylactic diet according to the metabolic test results. Accompanying anatomic abnormalities were seen low rates.

As a result, we can say that urolithiasis can be treated with the help of metaphylactic preventions and metabolic analysis in childhood.

\section{PO-0798 THE EVALUATION OF IMAGING TESTS IN RECURRENT URINARY TRACT INFECTIONS DURING CHILDHOOD}

${ }^{1} \mathrm{H}$ Gulsever Tosun, ${ }^{2} \mathrm{~A}$ Yazar, ${ }^{2} \mathrm{~S}$ Guven, ${ }^{2} \mathrm{~B}$ Karakayali, ${ }^{1} \mathrm{E}$ Pala. ${ }^{1}$ Family Medicine, Umraniye Training and Research Hospital, Istanbul, Turkey; ${ }^{2}$ Pedatrics, Umraniye Training and Research Hospital, Istanbul, Turkey

\subsection{6/archdischild-2014-307384.1433}

Background and aims Delay in diagnosis or insufficient treatment of recurrent urinary tract infections (UTI) may lead to hypertension, growth retardation, reflux nephropathy or chronic kidney failure. The aim of our study is to establish the incidence of urinary system anomaly at recurrent UTI and to compare the findings of urinary tract ultrasonography (USG), voiding cystourethrogram (VCUG) and Technetium-99 dimercaptosucsinic acid scintigraphy (DMSA).

Methods Our study included 92 children at age of 0 to 14 years old. Inclusion criteria's were: been diagnosed as UTI at least two times, had at least two positive urine culture and completed USG, VCUG ve Tc-99 m DMSA scintigraphy.

Results The mean age of subjects was $2.84 \pm 3.14$ years old (female/male ratio: 1.8/1). The mean age of VUR diagnosis was $3.31 \pm 2$ years with a female/male ratio of $2.6 / 1$. The renal scar has found at $27.2 \%$ in subjects with a diagnosis of VUR (23.9\%). Renal scar was significantly higher at subjects who had grade 3, 4 and 5 VUR, 9.7\% of subjects who specified normal by USG had a diagnosis of VUR by VCUG (sensitivity: 59\%, specifity: $87 \%$ ), $2.7 \%$ of subjects with normal VCUG were diagnosed as abnormal by DMSA (sensitivity: $72.7 \%$, specifity: $97.1 \%$, pozitive predictive value was $88.8 \%$ and negative predictive value was $91.89 \%$.

Conclusions VCUG and DMSA scintigraphy are very sensitive diagnostic tests at diagnosis of recurrent urinary tract infections. Using of these tests in children with recurrent urinary tract infections will prevent development of renal damage.

\section{PO-0799 BACTERIA ISOLATED FROM THE URINE SAMPLES OF CHILDREN AND THEIR ANTIBIOTIC SUSCEPTIBILITIES}

Y Taskiran. Çocuk, Aydin Kadin Dogum Hastanesi, Aydin, Turkey

\subsection{6/archdischild-2014-307384.1434}

The aim of this study was to identify the bacteria isolated from urine samples and to investigate their antibiotic suscep-tibilities in children aged $0-14$ years.

Urine samples of 1820 patients who referred to our hospital and had urinary tract infection symptoms were studied in order to evaluate culture and antibiotic susceptibility testing results. All urine samples were inoculated on eosin methylene blue (EMB) and $5 \%$ blood agars. Bacterial isolates were identified by conventional met-hods. Antibiotic susceptibility testing was performed by disk diffusion method.

The bacteria were isolated in 198 (9.2\%) of 1820 urine samples. The most frequently isolated bacteria (69.4\%) were Escherichia coli. Enterococcus spp. (12.7\%), Proteus spp. (6.1\%), Klebsiella spp. (4.3\%), Enterobacter spp. (5.9\%) and coagulasenegative staphylococci (CNS) (1.5\%) followed respectively. While all E.coli, Proteus spp., Klebsiella spp., and Enterobacter spp. isolates were susceptible to amikacin, gentamicin, and imipenem, sensitivity of the ampicillin (68.5\%), and the 